\title{
Comunicación de malas noticias en el ámbito prehospitalario
}

\section{Communication of bad news in the prehospital environment}

\author{
José Miguel Garrido-Molinaa \\ a Servicio de Emergencias 061 de Almería, Empresa Pública de Emergencias Sanitarias (EPES) Andalucía, España
}

\begin{abstract}
Resumen
Introducción: Los pacientes cada vez demandan más información. Dar información en el ámbito prehospitalario tiene dificultades añadidas. La comunicación eficaz de malas noticias ofrece a pacientes y familiares la posibilidad de mejorar el afrontamiento de situaciones difíciles. Objetivos: Analizar la evidencia científica disponible sobre la comunicación de malas noticias en el ámbito prehospitalario y determinar cómo influye en pacientes y familiares. Metodología: Se realizó una revisión bibliográfica donde se encontraron 8 artículos, los cuales cumplían los criterios de inclusión establecidos. Bases de datos consultadas: Pubmed, Cuiden Plus, Dialnet Plus y Lilacs. Resultados: La comunicación de malas noticias de una manera correcta permite a pacientes y familiares minimizar el dolor. Los profesionales sanitarios del ámbito prehospitalario demandan una formación y entrenamiento específico para estas situaciones. Conclusiones: La comunicación de malas noticias en el ámbito prehospitalario es necesaria e importante, tanto para pacientes como para familiares. El personal de los servicios de emergencia debe adquirir y entrenar los conocimientos necesarios para abordar la comunicación de malas noticias desde una nueva perspectiva ya que realizan su tarea diaria fuera de los centros sanitarios y en situaciones especiales.
\end{abstract}

Palabras clave: comunicación; malas noticias; prehospitalario; emergencia.

\begin{abstract}
Introduction: Patients increasingly demand more information. Giving information in the prehospital setting has additional difficulties. The effective communication of bad news offers patients and families the possibility of improving coping with difficult situations. Objective: Analyze the available scientific evidence on the communication of bad news in the prehospital setting and determine how it influences patients and families. Methodology: A bibliographic review was performed where 8 articles were found, which met the inclusion criteria previously established. Databases consulted: Pubmed, Cuiden Plus, Dialnet Plus and Lilacs. Results: The communication of bad news in a correct way allows patients and relatives to minimize pain. Health professionals in the prehospital setting demand specific training and training for these situations. Conclusions: The communication of bad news in the prehospital setting is necessary and important for both patients and relatives. The personnel of the emergency services must acquire and train the necessary knowledge to deal with the communication of bad news from a new perspective since they perform their daily tasks outside the health centers and in special situations.
\end{abstract}

Keywords: communication; bad news; prehospital; emergency. 


\section{Introducción}

$\mathrm{H}$

asta hace muy poco tiempo, la comunicación que mantenían los profesionales sanitarios con los pacientes y sus familiares ocupaba un mínimo papel de relevancia en el entorno sanitario, ya que primaban las competencias técnicas. En los últimos años, esta tendencia está cambiando debido a que los pacientes cada vez demandan más información. Además, atendiendo al modelo biopsicosocial como propuesta integradora, comprensiva y sistémica (Engel, 1977); los profesionales sanitarios han aumentado el interés en mejorar sus habilidades de comunicación con pacientes y familiares (Merayo, Bravo \& Gordón, 2014).

Desde una perspectiva sanitaria, podemos aceptar la definición de una mala noticia como, "La que va a cambiar de forma grave 0 adversa las perspectivas del paciente sobre su futuro" (Buckman, 1984).

Enfermería desempeña un papel esencial en la atención al paciente ante situaciones difíciles, su papel es muy amplio, abarcando desde el contacto físico e incluyendo el apoyo emocional, además, ayudan y apoyan a la familia durante el transcurso de este proceso (Mirón González, 2010; García-Rueda, Errasti-lbarrondo \& Arantzamendi, 2016; Maza, Zavala \& Escobar, 2009; Temel et al., 2017). Esto gana relevancia si tenemos en cuenta que la base del cuidado enfermero es ayudar a las personas mediante un enfoque holístico, encargándose de cubrir todas las dimensiones del cuidado (Gurdogan, Kurt, Aksoy, Kinici \& Şen, 2017).

En ciencias de la salud y en especial en el ámbito prehospitalario, donde a diario nos enfrentamos a situaciones de emergencia e incluso de catástrofes, el personal sanitario afronta multitud de situaciones difíciles, pero una de ellas es, sin duda, la de comunicar malas noticias tanto a pacientes como a familiares (Vazquez, Moya, Aponte, Nuño \& Beardo, 2015).

Proporcionar a los pacientes una información sencilla de comprender, les ofrece la oportunidad de ser partícipes en la toma de decisiones y, en muchos casos, disminuir el sufrimiento ante la comunicación de malas noticias (Mosqueda, Mendoza \& Jofré, 2014). La enfermería de urgencias y emergencias debería contar con habilidades específicas y entrenamientos adecuados para tal fin (Landa, López, Sánchez \& Jimenez, 2017), ya que recibir malas noticias puede provocar un déficit cognitivo, conductual o emocional en el receptor. Por todo ello, es imprescindible que los profesionales sanitarios que deben informar de una situación complicada realicen una comunicación efectiva (Ptacek \& Eberhardt, 1996).

\section{Objetivos}

- Identificar la evidencia científica disponible acerca de la comunicación de malas noticias en el ámbito prehospitalario.

- Determinar cómo influye en el paciente y en los familiares una adecuada comunicación de malas noticias dentro del ámbito prehospitalario.

\section{Metodología}

Se ha realizado una revisión bibliográfica de lo publicado en las bases de datos de Ciencias de la Salud entre Diciembre de 2018 y Febrero de 2019.

La búsqueda se llevó a cabo consultando los Descriptores de Ciencias de la Salud (DeCS) con las palabras clave: Comunicación (Communications), Malas noticias (Bad news), Prehospitalario (Prehospital), Emergencia (Emergencies). Además, también se consultaron los siguientes Medical Subject Headings (MeSH): Truth Disclosure, Emergency Medical Services y Health Communication. La estrategia de búsqueda se realizó a través de la combinación de las diferentes palabras clave y el uso de los operadores booleanos AND, NOT y OR.

Se establecieron como criterios de inclusión todos aquellos artículos que estuviesen relacionados con la comunicación de malas noticias en urgencias y emergencias. Se excluyeron los artículos que hacían referencia a este tema en otros ámbitos sanitarios.

En cuanto a los filtros utilizados, han sido los siguientes: Documentos publicados en los últimos 10 años (20102019), para incluir todos los artículos publicados sobre el tema; idioma inglés y castellano; y palabras clave contenidas en el título y en el resumen.

Una vez finalizada la búsqueda bibliográfica se inició el análisis de cada uno de los artículos escogidos. Un total de 8 artículos fueron seleccionados para formar parte de esta revisión, para ello se han seguido los criterios de inclusión. A continuación, en la Figura 1, se representan los resultados de la búsqueda bibliográfica mediante el diagrama de flujo PRISMA. 


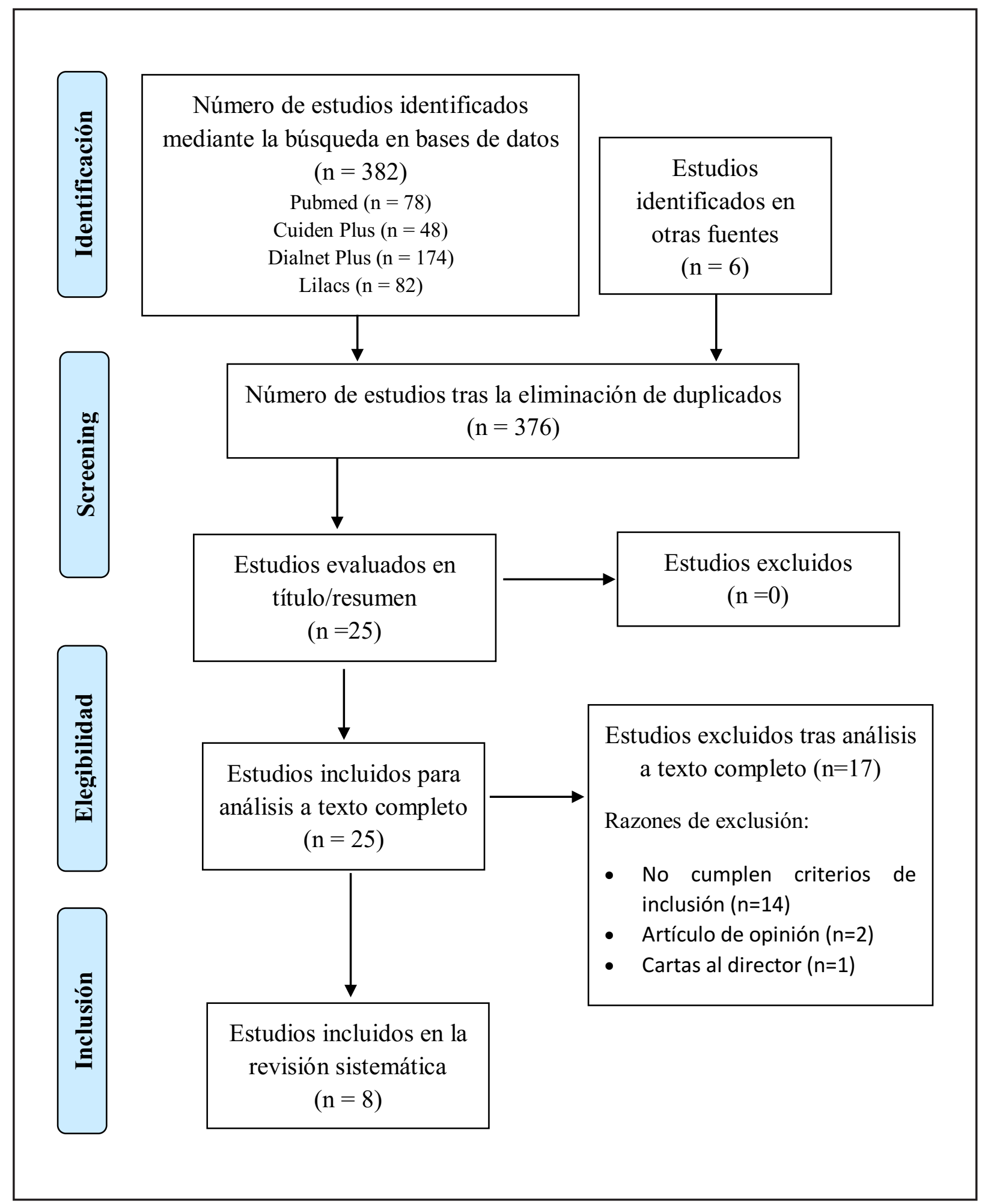

Figura 1: Bases de datos consultadas y resultados obtenidos. 


\section{Resultados y discusión}

Hemos encontrado estudios teóricos donde concluyen que los profesionales de Emergencias se encuentran con dificultades importantes a la hora de comunicar malas noticias. Entre otras, lo que obstaculiza esta comunicación, es tener que dar información de manera rápida, con el tiempo restringido para poder considerar aspectos éticos y probablemente existe una ausencia de conocimientos previos sobre lo acontecido y del historial del paciente. Además, en muchas ocasiones no se dispone del entorno adecuado para facilitar la información tanto a pacientes como a familiares, pero a pesar de ello, la información del estado y pronóstico del paciente se debe de dar cuanto antes (Rodríguez, 2014; Santos, Oliveira, Veronez, \& Nogueira, 2015; Erbay, Alan \& Kadioglu, 2013). Además, realizar la comunicación en el medio prehospitalario genera en los profesionales una situación estresante y desafiante (Douglas, Cheskes, Feldman \& Ratnapalan, 2012). Es significativo que el estrés y la incomodidad que generan este tipo de situaciones es inversamente proporcional a la experiencia profesional (Ferreira, Carvalho \& Cirino, 2017). Esto puede dejar en entredicho que en la formación académica este campo queda un poco vacío, adquiriéndose los conocimientos mediante la práctica clínica. Además, cuando se dan noticias en equipo, se lleva a cabo la comunicación de noticias difíciles de manera más humana y competente (Barlem et al., 2014).

Conocer la información sobre el diagnóstico y el pronóstico permite a los pacientes y familiares que el tiempo de espera transcurra con menos dolor (Rodriguez, 2014). Transmitir una información de calidad a la familia, puede mejorar el afrontamiento de la situación que atraviesan en esos momentos, disminuyendo la posibilidad de poner en peligro su salud física y mental, amortiguando las situaciones traumáticas que pueden arrojar muchísimo dolor (Santos et al., 2015).

Las peculiaridades que hacen diferente la atención sanitaria de emergencias respecto a otros cuidados en salud incluyen la necesidad de adquirir habilidades de comunicación. Se debería capacitar al personal en este campo, para que a la hora de intervenir se sientan menos estresados y más cómodos, aumentando la eficacia de la comunicación (Douglas et al., 2012; Erbay et al., 2013; Ferreira et al., 2017). Un entrenamiento precoz durante los estudios de pregrado puede potenciar el desarrollo de estas habilidades comunicadoras. El uso de actores 0 el entrenamiento entre los mismos estudiantes en situaciones de este tipo han demostrado la eficacia de estas estrategias (Luterberg, Graessel \& Sonath, 2014). Se podría incluir en los planes de estudios de pregrado una buena estrategia para la formación y adquisición de habilidades de comunicación de malas noticias en el ámbito prehospitalario. Por ejemplo, la gamificación está dando resultados prometedores al respecto (Boctor, 2013; Brull, Finlayson \& Kostelec, 2017; Johnsen, Fossum, Vivekananda-Schmidt, Fruhling \& Slettebø, 2018; Johnsen, Fossum, Vivekananda-Schmidt, Fruhling \& Slettebø, 2016; Milner \& Cosme, 2017). En la Tabla 1 se pueden observar resumidos los artículos seleccionados para esta revisión.

Una limitación del presente trabajo ha sido encontrar escasas publicaciones respecto a este tema, encontrándose principalmente estudios que incluyen a profesionales de medicina y enfermería.

Tabla 1. Resumen de artículos.

\begin{tabular}{|l|l|l|}
\hline \multicolumn{1}{|c|}{ Estudio } & \multicolumn{1}{|c|}{ Tipo de estudio } & \multicolumn{1}{c|}{ Resultados } \\
\hline $\begin{array}{l}\text { Barlem, E.L.D., Freitas, B.H., } \\
\text { Barlem, J.G.T., Ramos, A.M., } \\
\text { Oliveira, A.C.C., \& Piexak, D.R. } \\
\text { (2014). }\end{array}$ & $\begin{array}{l}\text { Estudio exploratorio descriptivo } \\
\text { de enfoque cualitativo }\end{array}$ & $\begin{array}{l}\text { Cuando se trabaja en equipo las } \\
\text { comunicaciones difíciles se realizan de una } \\
\text { manera más competente y humana. }\end{array}$ \\
\hline $\begin{array}{l}\text { Douglas, L., Cheskes, S., } \\
\text { Feldman, M. \& Ratnapalan, S. } \\
(2012) .\end{array}$ & $\begin{array}{l}\text { Estudio cualitativo descriptivo } \\
\text { basado en la teoría } \\
\text { fundamentada }\end{array}$ & $\begin{array}{l}\text { La notificación de una muerte es estresante } \\
\text { y desafiante. El estudio concluye que se } \\
\text { necesita tener formación específica para } \\
\text { comunicar malas noticias. }\end{array}$ \\
\hline $\begin{array}{l}\text { Erbay, H., Alan, S. \& Kadioglu, } \\
\text { S. (2013). }\end{array}$ & Estudio exploratorio descriptivo & $\begin{array}{l}\text { Los profesionales de la salud de } \\
\text { emergencias prehospitalarias suelen } \\
\text { dar información a pacientes y familiares } \\
\text { cuando la situación lo permite, pero ante } \\
\text { situaciones que amenazan la vida, dejan la } \\
\text { información en un segundo plano. }\end{array}$ \\
\hline
\end{tabular}


Tabla 1. Continuación.

\begin{tabular}{|l|l|l|}
\hline \multicolumn{1}{|c|}{ Estudio } & \multicolumn{1}{|c|}{ Tipo de estudio } & \multicolumn{1}{c|}{ Resultados } \\
\hline $\begin{array}{l}\text { Ferrerira da Silveira, F.J., } \\
\text { Carvalho Botelho C. \& Cirino } \\
\text { Valadão C. (2017). }\end{array}$ & $\begin{array}{l}\text { Estudio observacional } \\
\text { cuantitativo }\end{array}$ & $\begin{array}{l}\text { Los sanitarios con más años de } \\
\text { experiencia profesional se sienten } \\
\text { más cómodos y seguros a la hora } \\
\text { de transmitir malas noticias que los } \\
\text { menos experimentados. }\end{array}$ \\
\hline $\begin{array}{l}\text { Lutterberg, K., Graessel, E. \& } \\
\text { Simon, C. (2014). }\end{array}$ & $\begin{array}{l}\text { Estudio exploratorio } \\
\text { descriptivo }\end{array}$ & $\begin{array}{l}\text { Las habilidades de comunicación se } \\
\text { pueden enseñar en la etapa preclínica } \\
\text { aumentando su calidad en la práctica } \\
\text { clínica posterior. }\end{array}$ \\
\hline $\begin{array}{l}\text { Mosqueda-Diaz, A., Mendoza- } \\
\text { Parra, S. \& Jofré-Aravena, V. } \\
\text { (2014). }\end{array}$ & $\begin{array}{l}\text { Modelo de toma de decisiones } \\
\text { en salud de Ottawa }\end{array}$ & $\begin{array}{l}\text { El continuo contacto que desarrolla } \\
\text { enfermería con pacientes y familiares } \\
\text { hace que se encuentre en una } \\
\text { posición privilegiada para dar } \\
\text { iformación y promover la toma de } \\
\text { decisiones en salud. }\end{array}$ \\
\hline $\begin{array}{l}\text { Rodriguez, M. (2014). } \\
\text { Santos, L., Oliveira, L., Munari, } \\
\text { D., Barbosa, M., Peixoto, M., \& }\end{array}$ & descriptivo & $\begin{array}{l}\text { La comunicación e incluso al final } \\
\text { de la vida es una herramienta } \\
\text { importante. Tener información sobre } \\
\text { el diagnóstico y el pronóstico permite } \\
\text { a pacientes y familiares experimentar } \\
\text { este periodo con menos dolor. }\end{array}$ \\
\hline
\end{tabular}




\section{Conclusiones}

La comunicación de malas noticias en el ámbito prehospitalario es necesaria e importante tanto para pacientes como para familiares, ya que la falta de información genera efectos negativos sobre ellos. Sería importante que cada servicio de atención sanitaria contara con un protocolo específico y adaptado a sus necesidades para manejar esta información. Además, todo el personal sanitario debería estar formado y entrenado en este campo. Desde la enfermería de emergencias debemos de potenciar el cuidado holístico del paciente e intentar aliviar el sufrimiento, incluso cuando reciben malas noticias.

\section{Referencias Bibliográficas}

Barlem, E.L.D., Freitas, B.H., Barlem, J.G.T, Ramos, A.M., Oliveira, A.C.C., \& Piexak, P. D. R. (2014). Comunicação de notícias difíceis em uma Unidade de Tratamento Intensivo Neonatal. Revista de Enfermagem, 8(7), 1853-1859. Doi: https://doi. org/10.5205/reuol.5963-51246-1-RV.0807201404

Boctor, L. (2013). Active-learning strategies: The use of a game to reinforce learning in nursing education. A case study. Nurse Education in Practice, 13(2), 96-100. Doi: https://doi.org/10.1016/j. nepr.2012.07.010

Brull, S., Finlayson, S., Kostelec, T., Macdonald, R., \& Krenzischeck, D. (2017). Using Gamification to Improve Productivity and Increase Knowledge Retention during Orientation. Journal of Nursing Administration, 47(9), 448-453. Doi: https://doi. org/10.1097/NNA.0000000000000512

Buckman, R. (1984). Breaking bad news: Why is it still so difficult? British Medical Journal, 288, 1597-1599. https://doi.org/10.1177/002205740305801214

Douglas, L., Cheskes, S., Feldman, M., \& Ratnapalan, S. (2012). Paramedics'experiences with death notification: a qualitative study. Journal of Paramedic Practice, 4(9), 533-539.

Engel, G. (1977). The need for a new medical model: a challenge for biomedicine. Science, 196, 129-136.

Erbay, H., Alan, S., \& Kadioglu, S. (2014). Attitudes of prehospital emergency care professionals toward refusal of treatment: A regional survey in Turkey. Nursing Ethics, 21(5), 530-539. Doi: https://doi. org/10.1177/0969733013505311

Ferreira da Silveira, F. J., Carvalho Botelho, C., \& Cirino
Valadão, C. (2017). Breaking bad news: doctors' skills in communicating with patients. Sao Paulo Medical Journal, 135(4), 323-331. Doi: https://doi. org/10.1590/1516-3180.20160221270117

García-Rueda, N., Errasti-Ibarrondo, B., \& Arantzamendi Solabarrieta, M. (2016). La relación enfermerapaciente con enfermedad avanzada y terminal: revisión bibliográfica y análisis conceptual. Medicina Paliativa, 23(3), 141-152. doi: 10.1016/j. medipa.2014.01.003

Gurdogan, E., Kurt, D., Aksoy, B., Kınıcı, E., \& Şen, A. (2017). Nurses' perceptions of spiritual care and attitudes toward the principles of dying with dignity: A sample from Turkey. Death Studies, 41(3), 180187. doi: $10.1080 / 07481187.2016 .1231242$

Johnsen H., Fossum M., Vivekananda-Schmidt, P., Fruhling A., \& Slettebo, A. (2016). Teaching clinical reasoning and decisión-making skills to nursing students: Desing, development and usability evaluation of a serious game. International Journal Medical Informatics, 94, 39-48. Doi: 10.1016/ j.jjmedinf.2016.06.014

Johnsen, H. M., Fossum, M., Vivekananda-Schmidt, P., Fruhling, A., \& Slettebø, Å. (2018). Developing a serious game for nurse education. Journal of Gerontological Nursing, 44(1), 15-19. https://doi. org/10.3928/00989134-20171213-05

Landa-Ramírez, E., López-Gómez, A., Jiménez-Escobar, I., \& Sánchez-Sosa, J. (2017). Comunicación de malas noticias en urgencias médicas: recomendaciones y retos futuros. Revista Médica del Instituto Mexicano del Seguro Social, 55(6), 736-47.

Luttenberger, K., Graessel, E., Simon, C., \& Donath, C. (2014). From board to bedside - training the communication competences of medical students with role plays. BMC Medical Education, 14(1), 1-10. Doi: https://doi.org/10.1186/1472-6920-14-135

Maza Cabrera, M. M., Zavala Gutiérrez, M. Z., \& Escobar, J. M. M. (2009). Actitud del profesional de enfermeria ante la muerte de pacientes. Ciencia y Enfermeria, 15(1), 39-48. Doi: https://doi.org/doi: 10.4067/s071795532009000100006

Merayo, A., Bravo, E., \& Gordón, F. (2014). La comunicación con el paciente. Habilidades emocionales para los profesionales de la salud. Barcelona, España: Elsevier.

Milner, K. A., \& Cosme, S. (2017). The PICO Game: An 
Innovative Strategy for Teaching Step 1 in EvidenceBased Practice. Worldviews on Evidence-Based Nursing, 14(6), 514-516. https://doi.org/10.1111/ wvn. 12255

Mirón González, R. (2010). Comunicación de malas noticias: perspectiva enfermera. Revista Española de Comunicación en Salud, 1(1), 39-49.

Mosqueda-Diaz, A., Mendoza-Parra, S., \& JofréAravena, V. (2014). Aporte de enfermería a la toma de decisiones en salud. Revista Brasileira de Enfermagem, 67(3), 462-67.

Ptacek, J., \& Eberhardt, T. (1996). Breaking bad news. A review of the literature. JAMA - Journal of the American Medical Association, 276(6), 496502. doi: https://doi.org/10.1111/j.1600-0447.1960. tb08330.x

Rodriguez, M. (2014). Despedida silenciada: Equipe médica, família, paciente-cúmplices da conspiração do silêncio. Psicologia Revista, 23(2), 261-272.

Santos, L. F., Oliveira, L. M., Munari, D. B., Barbosa, M. A., Peixoto, M., \& Nogueira, A. (2015). When the communication is harmful in the encounter between health professional and family of hospitalized child. Enfermería Global, 14(37), 216-226.

Temel, J., Greer, J., El-Jawahri, A., Pirl, W., Park, E., Jackson, V.A. ... Ryan, D.P. (2017). Effects of Early Integrated Palliative Care in Patients With Lung and GI Cancer: A Randomized Clinical Trial. Journal Of Clinical Oncology, 35(8), 834-841. doi: 10.1200/ jco.2016.70.5046

Vázquez González, A., Moya Meléndez R., Aponte Tomillo I., Nuño Morales P., \& Beardo Rodríguez G. (2015). Actuación de enfermería ante las malas noticias en urgencias. Revista Enfermería Docente, (103), 33-37. 